\title{
Chromaticity and wakefield effect on the transverse motion of longitudinal bunch slices in the Fermilab Tevatron
}

\author{
V.H. Ranjbar \\ Tech-X Corporation, 5621 Arapahoe Avenue, Suite A, Boulder, Colorado 80303, USA \\ P. Ivanov* \\ Fermilab, P.O. Box 500 Batavia, Illinois 60510-5011, USA \\ (Received 15 November 2007; published 13 August 2008)
}

\begin{abstract}
The transverse turn-by-turn evolution of a bunch slice after an impulse kick is examined considering chromatic and impedance effects. It is found that by fitting the envelope of the beam slice motion to simulated data the strength of the resistive wall wakefield can be determined.
\end{abstract}

DOI: 10.1103/PhysRevSTAB.11.084401

PACS numbers: 29.27.Bd, 29.27.Fh

\section{INTRODUCTION}

The Tevatron Run II project seeks to deliver $1.96 \mathrm{TeV}$ center of mass proton-antiproton collisions to experiments CDF and D0 located at two collision points in the Tevatron ring. The process begins with the production of protons which are accelerated through four stages beginning with a $400 \mathrm{MeV}$ linac, $8 \mathrm{GeV}$ Booster, $150 \mathrm{GeV}$ main injector, and final acceleration in the Tevatron to $980 \mathrm{GeV}$. Prior to this protons are extracted from the main injector and fired against a tungsten target to produce antiprotons which are then transported and cooled in the debuncher and further cooled and accumulated in the accumulator and recycler. After enough cooled antiprotons have been accumulated for a Tevatron store, both species are then accelerated to collision energy of $980 \mathrm{GeV}$.

In order to deliver as many collisions as possible to the experiments, nonluminous proton and antiproton losses need to be reduced as much as possible. One significant source of continuous loss is related to head-tail instabilities driven by wakefields. These instabilities require running the Tevatron with a large chromaticity at high energy physics store. High chromaticities, however, increase the total tune spread and reduce beam lifetime by increasing the likelihood that a particle will cross a resonant tune value and either be lost or have its action increased.

Clearly an understanding of this head-tail instability and the evolution of the bunch is important if we seek to run the Tevatron with a lower chromaticity and ways to counteract losses are sought. Improvements in the speed of available digital oscilloscopes now make it possible to experimentally measure the transverse evolution of longitudinal bunch slices down to resolutions below $0.4 \mathrm{~ns}$. It is now possible to directly compare existing or new models with experiment and arrive at a more exact characterization of the collective transverse motion of a single coalesced bunch in the Tevatron.

\footnotetext{
*Presently at Budker Institute of Nuclear Physics, Novosibirsk, Russia.
}

As a result of previous efforts to understand the source and magnitude of the impedance in the Tevatron, improvements have been made to bring down the effective impedance from $5 \mathrm{M} \Omega / \mathrm{m}$ to now $1 \mathrm{M} \Omega / \mathrm{m}[1,2]$. This work was based on the analysis of data obtained from this new breed of fast digital oscilloscopes using primarily the instability growth rate as compared with simulation.

In this paper we present a systematic approach where a careful comparison between simulation and measurement allows one to fit the transverse wakefield by fitting the observable deformation of the transverse bunch profiles. This allows for the development of a new approach to estimate the strength and likely structure of the short range wakefield by comparison with a model with only three relevant parameters. Two of these parameters, linear and 2nd order chromaticity, are independently measurable leaving only the strength and structure of the wakefield to be determined from a fit.

\section{EXPERIMENTAL SETUP}

Using the $1 \mathrm{~m}$ long stripline detector installed at F0 in the Tevatron, the signal was captured using a Tektronixs Digital Phosphorus TDS7154 oscilloscope. The $A-B$ and $A+B$ signals were measured in fast-frame mode with a resolution of $0.4 \mathrm{~ns}$ across an $8 \mathrm{~ns}$ frame for 1000-2000 turns. The setup can be seen in Fig. 1. The signal from the plates consists of the sum of the image current traveling with the beam and the reflected image of the beam at $2 L / c$ ( $L$ is the stripline length) from the downstream end [3]. We reconstructed the single image by subtracting out the reflected image by splitting the signal and delaying and resumming a copy of the signal by $2 L / c$. Reconstruction can be accomplished either digitally or in an analog fashion by delaying and resumming the signals in the appropriate manner. After reconstruction of the signal, the transverse position could then be evaluated using

$$
Z(n, \tau)=E \frac{[A(n, \tau)-B(n, \tau)]}{[A(n, \tau)+B(n, \tau)]} .
$$




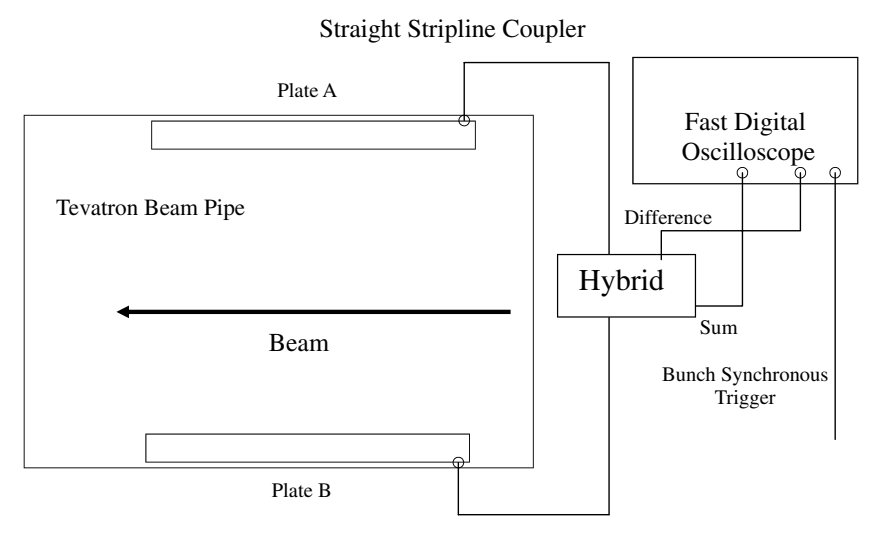

FIG. 1. Experimental setup.

Here $E$ is a geometric factor from the angular width of the stripline, $n$ the turn number, and $\tau$ the longitudinal slice.

\section{COMPARISON WITH A SIMPLE ANALYTICAL MODEL}

In the simplest case, a single particle's transverse motion can be characterized by

$$
\frac{d^{2} Y(s)}{d s^{2}}+\omega_{\beta}^{2} / c^{2} Y(s)=0 .
$$

Here $Y$ is the transverse position of the particle, $s$ is the longitudinal coordinate, and $\omega_{\beta}$ gives the angular betatron frequency, which is just the angular revolution frequency $\omega_{0}$ times $Q$ the betatron tune. Solutions give transverse harmonic motion oscillating with the betatron tune $Q$ each revolution. However, if the particle resides in a rf bucket, one must consider its longitudinal motion inside of the bucket as well and the equation of motion becomes

$$
\frac{d^{2} Y(s, \delta, z)}{d s^{2}}+\omega_{\beta}^{2}(\delta) / c^{2} Y(s, \delta, z)=0 .
$$

Here $z$ defines the longitudinal position relative to the center of the rf bucket, and $\delta=\Delta p / p$ the relative momentum difference from the "on momentum" particle. If we expand the betatron frequency to first order in $\delta$, we obtain

$$
\omega_{\beta}(\delta)=\omega_{0} Q+\xi \omega_{0} \delta .
$$

Here $\xi=\frac{d Q}{d \delta}$ is called the chromaticity. We can also approximate the longitudinal motion inside the rf bucket using

$$
\begin{aligned}
& \delta(s)=\frac{-\omega_{s}}{\eta c} r \sin \left(\omega_{s} s / c+\phi\right) \\
& z(s)=r \cos \left(\omega_{s} s / c+\phi\right) .
\end{aligned}
$$

Here $\omega_{s}=\omega_{0} Q_{s}$ known as the synchrotron angular frequency is the angular frequency of the longitudinal motion, and $\phi$ is the phase of the synchrotron motion, $\eta$ is the phase-slip factor defined as the fractional change of the revolution period per unit of $\delta$. We have also defined $r^{2}=z(s)^{2}+\left(\frac{\eta c}{\omega_{s}}\right)^{2} \delta(s)^{2}$. form

Following [4], an approximate solution to Eq. (3) has the

$$
Y(s, \delta, z) \approx A e^{ \pm i \Phi(s, \delta, z)},
$$

where $A$ is the constant amplitude and

$$
\begin{aligned}
\Phi(s, \delta, z)= & \int_{0}^{s} d s^{\prime}\left[\omega_{0} Q / c+\omega_{0} \xi \delta(s) / c\right] \\
\Phi(s, \delta, z)= & \omega_{0} Q s / c+\frac{\xi \omega_{0}}{\eta c}[z(s)-z(0)] \\
\Phi(s, \delta, z)= & \omega_{0} Q s / c+\frac{\xi \omega_{0} z}{\eta c}\left[1-\cos \left(\omega_{s} s / c\right)\right] \\
& +\frac{\xi \delta}{Q_{s}} \sin \left(\omega_{s} s / c\right) .
\end{aligned}
$$

Here it is assumed that the particles are kicked together. As a result they are all in phase with $\Phi(0, \delta, z)=0 . z(0)$ is now recast in terms of $z(s)$ and $\delta(s)$ using the transformation from the frame rotating with the synchrotron frequency:

$$
\left(\begin{array}{l}
z(s) \\
\delta(s)
\end{array}\right)=\left(\begin{array}{cc}
\cos \left(\omega_{s} s / c\right) & \frac{\eta c}{\omega_{s}} \sin \left(\omega_{s} s / c\right) \\
-\frac{\omega_{s}}{\eta c} \sin \left(\omega_{s} s / c\right) & \cos \left(\omega_{s} s / c\right)
\end{array}\right)\left(\begin{array}{c}
z(0) \\
\delta(0)
\end{array}\right) .
$$

If we integrate $Y$ over a Gaussian $\delta$ distribution $\rho(\delta)=$ $e^{-\left[\delta^{2} /\left(2 \sigma_{\delta}^{2}\right)\right]} /\left(\sqrt{2} \pi \sigma_{\delta}\right)$, we can obtain an expression for the transverse motion for a "fixed" longitudinal slice

$$
\begin{aligned}
Z(s, \tau)= & \operatorname{Re} \int_{-\infty}^{\infty} d \delta \rho(\delta) Y(s, \delta, z)=e^{-\left(\chi^{2} / 2\right) \sigma_{\tau}^{2} \sin ^{2}\left(\omega_{s} s / c\right)} \\
& \times \sin \left\{\omega_{0} Q s / c+\chi \tau\left[1-\cos \left(\omega_{s} s / c\right)\right]\right\}
\end{aligned}
$$

where we have defined a head-tail phase factor $\chi=\frac{\omega_{0} \xi}{\eta}$ and set $z=\tau c$ and assuming the $\tau$ and $\delta$ distributions are matched to the rf bucket we can use, $\sigma_{\delta}=\frac{\omega_{s} \sigma_{\tau}}{|\eta|}$, where $\sigma_{\tau}$ is the rms bunch longitudinal length in time. As can be seen in Fig. 2, the result yields collective motion with an envelope which decoheres and recoheres every $1 / 2$ synchrotron period and a phase which decoheres and recoheres every synchrotron period.

Comparing this simple model against measured data from the bunch center (see Fig. 3) shows a similar coherence-decoherence pattern, with the strength of decoherence correlating with the chromaticity. While this simple model captures many of the features of the transverse collective motion of the beam, there are many other aspects of the motion which even a cursory comparison shows to be deficient. A comparison of the model with the actual beam motion in Fig. 4 reveals a difference in long-term decoherence and $\tau$ dependence. 

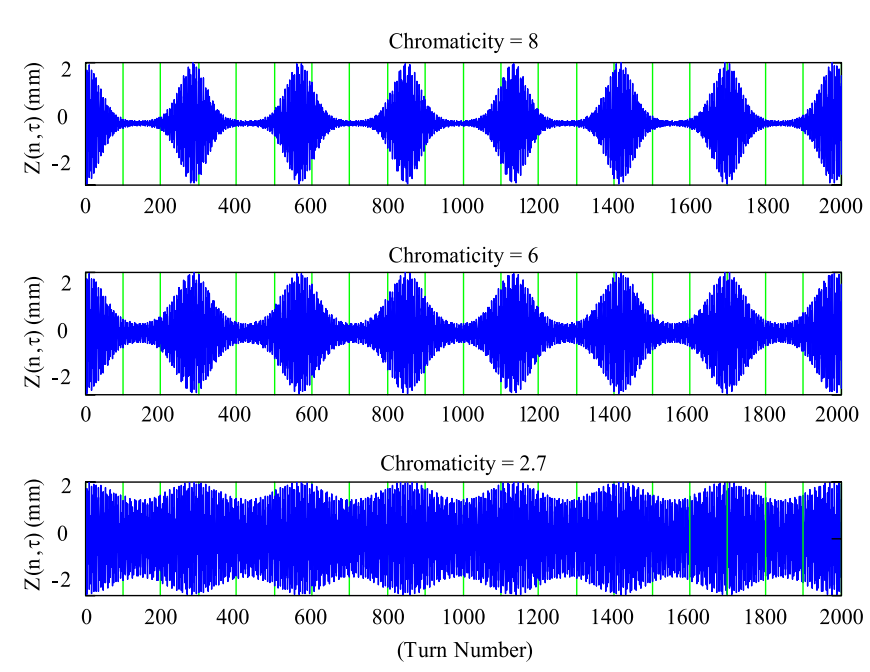

FIG. 2. (Color) Plot of $Z(n, \tau)$ simulated versus $n$ turns with $\tau=$ 0 (bunch center). Shown is the analytical model of transverse beam slice motion after $1 \mathrm{~mm}$ kick. With fixed rms bunch length $\sigma_{\tau}=3 \mathrm{~ns}$ and decreasing chromaticity, we can see the depth of the decoherence envelope decrease.

Without too much difficulty this analysis can be taken a step further to include the effects of 2 nd order chromaticity $\xi^{\prime}$ again following [4], the betatron tune can be expanded to second order in $\delta$ :

$$
\omega_{\beta}(\delta)=\omega_{0}\left(Q+\xi \delta+\frac{1}{2} \xi^{\prime} \delta^{2}\right)
$$

The phase $\Phi(s, \delta, z)$ can be found by including the second order terms in the integral in Eq. (7). The result is the addition of three terms to $\Phi$ :
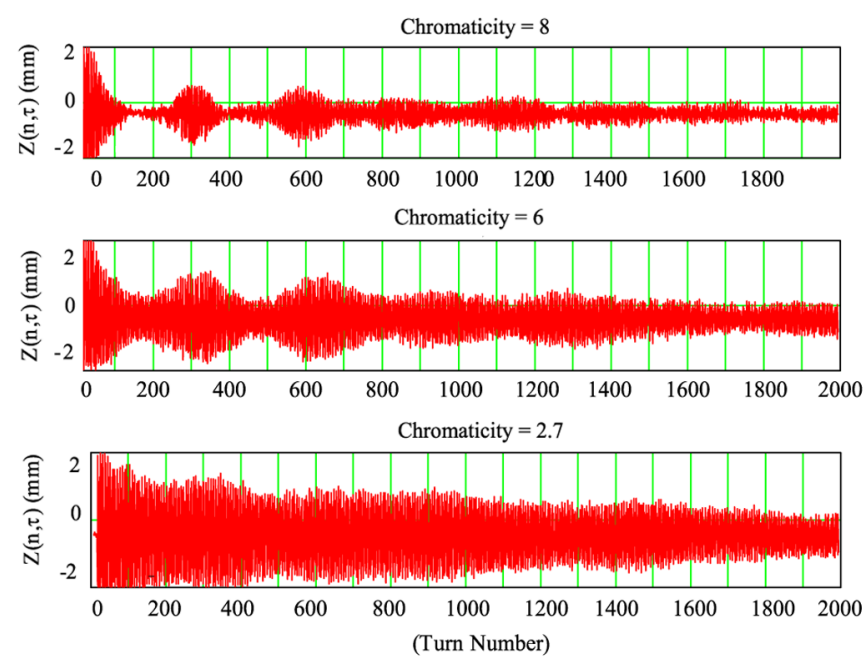

FIG. 3. (Color) Plot of $Z(n, \tau)$ measured versus $n$ turns with $\tau=$ 0 (bunch center). Shown is measured data of the transverse beam slice motion after $1 \mathrm{~mm}$ kick. With fixed rms bunch length $\sigma_{\tau}=$ 3 ns and decreasing chromaticity, we can see the depth of the decoherence envelope decrease, comparable to simple analytical model.
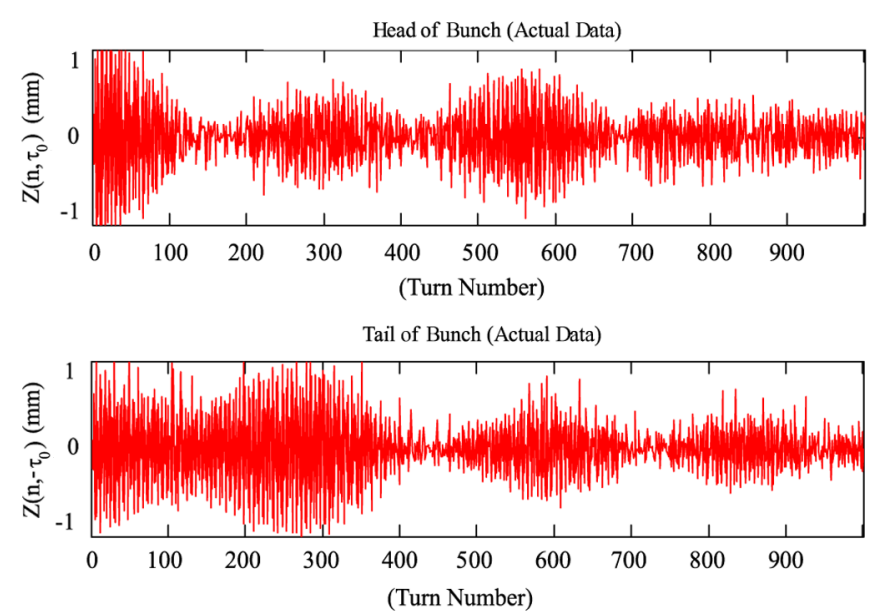

FIG. 4. (Color) Plot of $Z(n, \tau)$ measured versus $n$ turns. Shown is the transverse beam motion after $1 \mathrm{~mm}$ kick with $0.4 \mathrm{nsec}$ longitudinal bunch slices of head (top) and the tail (bottom) each 4 nsecs from the bucket center $\left(\tau_{0}=4\right.$ nsecs $)$. Chromaticity measured to be $\xi=4.0 \pm 1$ and $\sigma_{\tau}=3$ nsecs.

$$
\begin{aligned}
& \frac{\omega_{0} \xi^{\prime} \tau \delta}{4 \eta}\left[1-\cos \left(2 \omega_{s} s / c\right)\right]+\frac{\omega_{0} \omega_{s}^{2} \xi^{\prime} \tau^{2}}{4 \eta^{2}}(s / c \\
& \left.-\frac{\sin \left(2 \omega_{s} s / c\right)}{2 \omega_{s}}\right)+\frac{\omega_{0} \xi^{\prime} \delta^{2}}{4}\left(s / c+\frac{\sin \left(2 \omega_{s} s / c\right)}{2 \omega_{s}}\right) .
\end{aligned}
$$

As with the first order case, this equation can also be integrated over a Gaussian distribution analytically yielding

$$
\begin{aligned}
\alpha(n, \tau)= & \left(\frac{\xi}{Q_{s}} \sin \left(2 n \pi Q_{s}\right)+\frac{\omega_{0} \xi^{\prime}}{4 \eta} \tau\left[1-\cos \left(4 n \pi Q_{s}\right)\right]\right) \sigma_{\delta} \\
\theta(n)= & \pi \xi^{\prime} \sigma_{\delta}^{2}\left(n+\frac{\sin \left(4 n \pi Q_{s}\right)}{4 \pi Q_{s}}\right) \\
\phi(n, \tau)= & \frac{\omega_{0} \xi}{\eta} \tau\left[1-\cos \left(2 n \pi Q_{s}\right)\right] \\
& +\frac{\pi \omega_{0}^{2} Q_{s}^{2} \xi^{\prime}}{2 \eta^{2}} \tau^{2}\left(n-\frac{\sin \left(4 n \pi Q_{s}\right)}{4 \pi Q_{s}}\right) \\
& -\frac{1}{2}\left(\frac{\alpha^{2}(\tau, n) \theta(n)}{1+\theta^{2}(n)}-\arctan [\theta(n)]\right) \\
Z(n, \tau)= & \frac{e^{\left[\alpha(n, \tau)^{2}\right] /\left\{2\left[1+\theta(n)^{2}\right]\right\}}}{\left.\left[1+\theta(n)^{2}\right)\right]^{1 / 4}} \sin [2 n \pi Q+\phi(n, \tau)] .
\end{aligned}
$$

Here the time variable is rescaled, setting $s=n C$, where $C$ is the circumference of the ring and $n$ is the turn number. The inclusion of 2 nd order chromaticity shown in Fig. 5 reveals that we can capture the long-term decoherence and some aspects of the beam envelope's $\tau$ dependence. However, we cannot explain the coherence-recoherence, pattern, where a small recoherence is followed by a large recoherence. Since the remaining outstanding effect operates asymmetrically on the bunch, we believe that this should be explained by the inclusion of wakefields. Other 


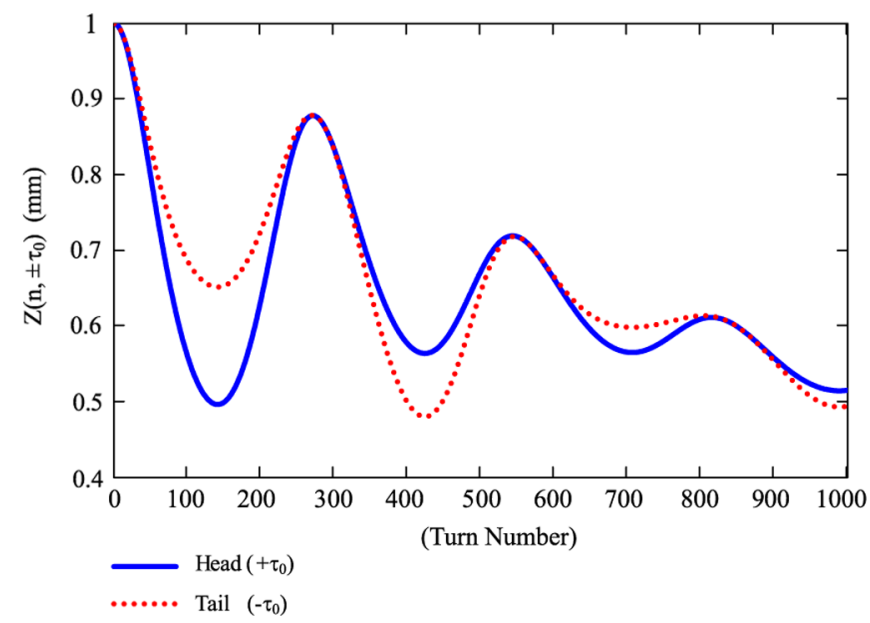

FIG. 5. (Color) Plot of the envelope of $Z(n, \tau)$ from Eq. (12). After $1 \mathrm{~mm}$ kick with $0.4 \mathrm{~ns}$ longitudinal bunch slices of head (solid trace) and the tail (dotted) each 4 nsecs $\left(\tau_{0}=4\right.$ nsecs) from the bucket center. Chromaticity is set to be $\xi=4.0, \xi^{\prime}=$ 1500 , and $\sigma_{\tau}=3$ nsecs.

effects, such as space charge and synchrotron tune spread, representing tune shifts on the order of $7 \times 10^{-4}$, operate symmetrically on the bunch and therefore could not account for the behavior of the beam envelope.

As can be see in Figs. 6 and 7, this structural effect depends strongly on bunch intensity, which is another clear sign that it is caused by wakefields.

In this paper we will examine the effect of transverse wakefields and construct a model which combines the effects of linear, 2nd order chromaticity, and resistive
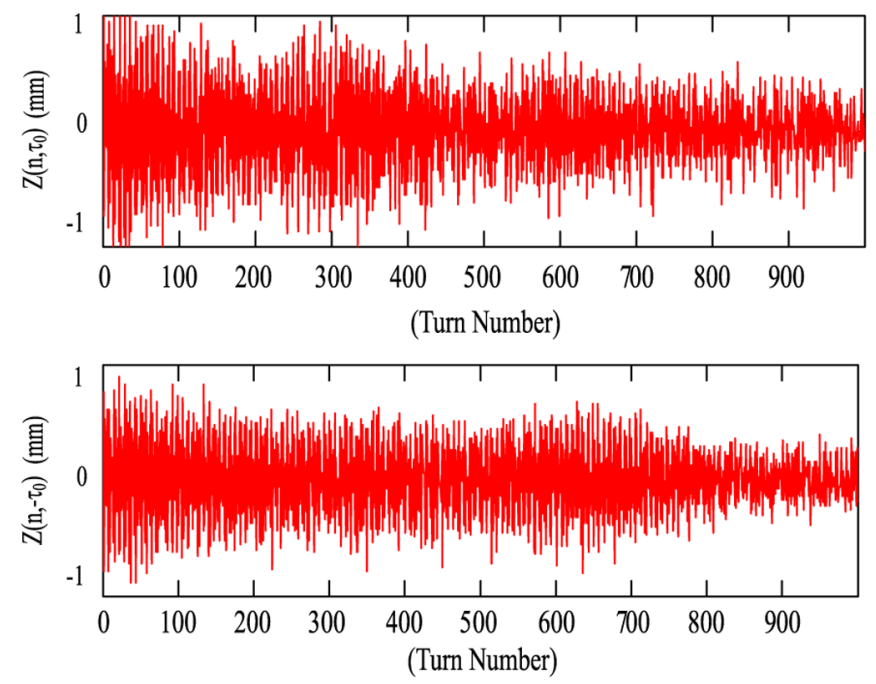

FIG. 6. (Color) Plot of $Z(n, \tau)$ measured versus $n$ turns. Shown is a low intensity bunch with $N_{p}=0.9 \times 10^{11}$. The transverse beam motion after $1 \mathrm{~mm}$ kick with $0.4 \mathrm{nsec}$ longitudinal bunch slices of head (top) and the tail (bottom) each 4 nsecs $\left(\tau_{0}=\right.$ 4 nsecs) from the bucket center. Chromaticity measured to be $\xi=4.0 \pm 1$ and $\sigma_{\tau}=3$ nsecs.
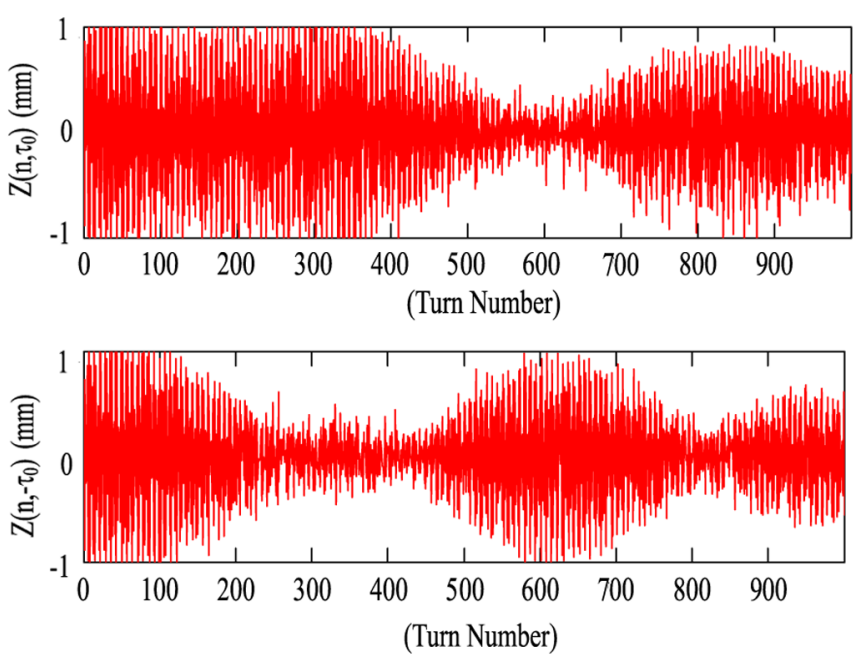

FIG. 7. (Color) Plot of $Z(n, \tau)$ measured versus $n$ turns. Shown is a high intensity bunch with $N_{p}=2.7 \times 10^{11}$. Transverse beam motion after $1 \mathrm{~mm}$ kick with $0.4 \mathrm{nsec}$ longitudinal bunch slices of head (top) and the tail (bottom) each $4 \operatorname{nsecs}\left(\tau_{0}=\right.$ 4 nsecs) from the bucket center. Chromaticity is measured to be $\xi=4.0 \pm 1$ and $\sigma_{\tau}=3$ nsecs.

wall transverse wakefields. This model reflects the dominant behavior of the transverse collective motion of a single coalesced bunch in the Tevatron.

\section{THE EFFECT OF TRANSVERSE WAKEFIELDS}

The inclusion of transverse wakefields in Eq. (3) requires the addition of a term on the right-hand side which is the integral of the transverse motion of all particles "ahead" of the current particle [5],

$$
-\frac{r_{0}}{C \gamma} \int_{-\infty}^{\infty} \int_{z}^{\infty} d \delta d z^{\prime} \rho\left(z^{\prime}, \delta\right) Y\left(s, \delta, z^{\prime}\right) W_{\perp}\left(z-z^{\prime}\right) .
$$

Here $r_{0}=e^{2} / m_{0} c^{2}, C$ is the circumference of the ring, $W_{\perp}(z)$ is the transverse wakefield, $N=\int d z^{\prime} \rho\left(z^{\prime}\right)$ is the number of particles in a bunch.

We can recast the differential equation with the wakefield driving term by using the solution we already found for the homogenous equation $Y=A \exp \left\{-i \frac{\chi}{c} \times\right.$ $\left.[z(s)-z(0)]+i \omega_{\beta} s / c\right\}$, again dropping all terms of the order $Q_{s}^{2}$ which includes $A^{\prime \prime}$ since the amplitude oscillations are observed to be on the order of $Q_{s}$, we can obtain a first order differential-integral equation,

$$
\begin{aligned}
\frac{d A}{d s}= & i \alpha e^{i(\chi / c) z\left[1-\cos \left(\omega_{s} s / c\right)\right]+i \delta\left(\xi / Q_{s}\right) \sin \left(\omega_{s} s / c\right)} \\
& \times \int_{-\infty}^{\infty} \int_{z}^{\infty} d \delta^{\prime} d z^{\prime} \rho\left(z^{\prime}, \delta^{\prime}\right) A\left(s, \delta^{\prime}, z^{\prime}\right) \\
& \times e^{-i(\chi / c) z^{\prime}\left[1-\cos \left(\omega_{s} s / c\right)\right]-i \delta^{\prime}\left(\xi / Q_{s}\right) \sin \left(\omega_{s} s / c\right)} W_{0 \perp}\left(z-z^{\prime}\right),
\end{aligned}
$$

setting $\alpha=\frac{r_{0} W_{1 \perp} N c}{2 \omega_{\beta} \gamma C}$ and using $\tau=z / c$. We have factored 
out the $W_{1 \perp}$ which we define as the constant coefficient of the transverse wakefield function $W_{\perp}=W_{1 \perp} W_{0 \perp}(z)$. This equation can be numerically integrated for any given number of macroparticles $N_{p}$, wakefield and initial distribution. This treatment neglects amplitude dependent effects since the measurements considered were taken in the absence of powered octupoles and previous experiments and models $[1,2,6]$ indicate a negligible amplitude dependent effect at the oscillation amplitudes our measurements were taken.

We use a simple Euler step integrator method and step the amplitude using

$$
\begin{aligned}
d A\left[z_{i p 1}(n), n\right]= & i w_{r} \sum_{i p 2}^{N_{p}} A\left[z_{i p 2}(n), n\right] \\
& \times e^{(-i \chi / c)\left[z_{i p 1}(n)-z_{i p 1}(0)-z_{i p 2}(n)+z_{i p 2}(0)\right]} \\
& \times W_{0 \perp}\left[z_{i p 1}(n)-z_{i p 2}(n)\right] .
\end{aligned}
$$

Here the wakefield is causal with $W_{0 \perp}(x)=0$ when $x>0$ and $z_{i}(n)=r_{i} \cos \left[\phi_{i}(n)\right]$ and the phase $\phi(n)$ is the synchrotron phase. A random Rayleigh distribution in $r_{i}$ and uniform in $\phi(0)_{i}$ for $N_{p}$ particles is used to develop a Gaussian distribution in $\tau$ and $\delta$. We have also introduced $w_{r}$ which is just $w_{r}=\alpha \frac{C}{Q_{s} \sqrt{\sigma_{z}} N_{p}}$, which is a combination of our integration time step, normalization, and wake strength. At the end of each step, we integrate over $\delta$,

$$
\begin{aligned}
A b(z, n)= & \sum_{i p}^{N_{p}} A\left[z_{i p}(n), n\right] e^{(-i \chi / c)\left[z_{i p}(n)-z_{i p}(0)\right]} \\
& \times \Theta\left[\left|z-z_{i p}(n)\right|-L\right],
\end{aligned}
$$

where $L$ is the pickup length and $\Theta(x)$ is the step function.
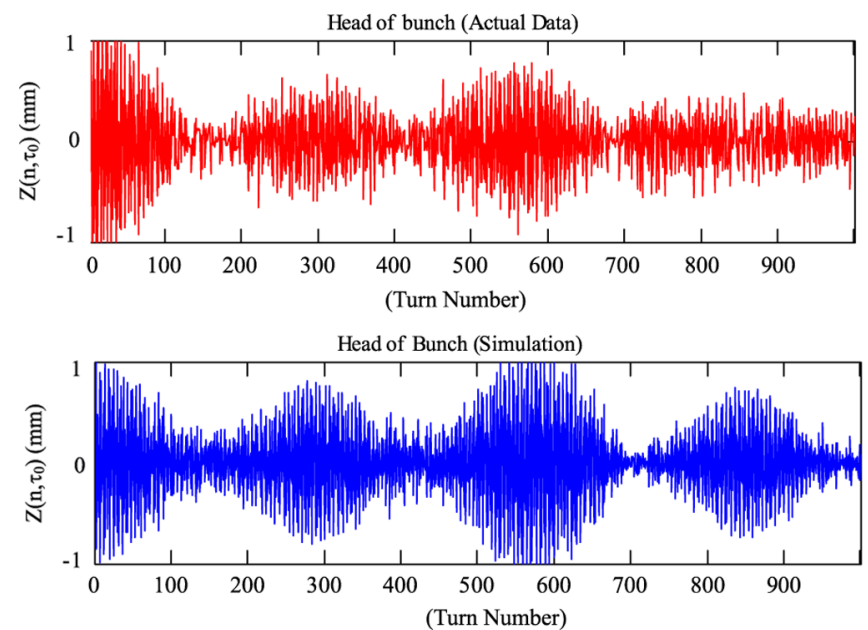

FIG. 8. (Color) Plot of measured and simulated $Z(n, \tau)$ versus $n$ turns. Shown is the transverse (horizontal) turn-by-turn data of head after a $1 \mathrm{~mm}$ kick 4 nsecs ahead of bucket center $\left(\tau_{0}=\right.$ 4 nsecs). Actual turn-by-turn data (top) compared with 1000 particle simulation with resistive wall wake $W_{1 \perp}=12 \mathrm{~cm}^{1.5}$ and chromaticity $\xi=3.733$ and $\sigma_{\tau}=3$ nsecs.
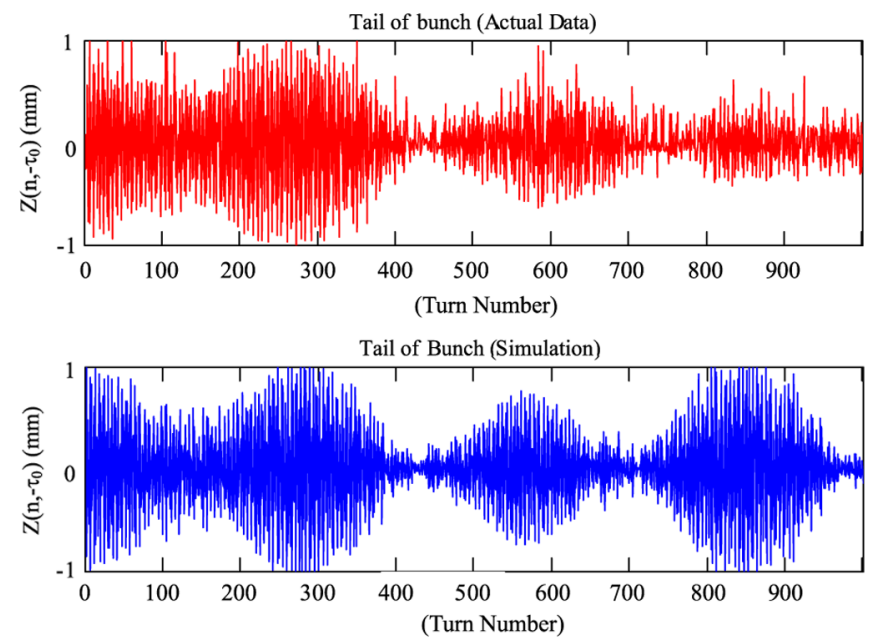

FIG. 9. (Color) Plot of measured and simulated $Z(n, \tau)$ versus $n$ turns. Shown is the transverse (horizontal) turn-by-turn data of tail after a $1 \mathrm{~mm}$ kick 4 nsecs behind bucket center $\left(\tau_{0}=\right.$ 4 nsecs). Actual turn-by-turn data (top) compared with 1000 particle simulation with resistive wall wake $W_{1 \perp}=12 \mathrm{~cm}^{1.5}$ and chromaticity $\xi=3.733$ and $\sigma_{\tau}=3$ nsecs.

We begin with Gaussian distribution of $N_{p}$ particles in $z$. Using a resistive wall wakefield of the form [7],

$$
W_{0 \perp}(z)=\frac{1}{\sqrt{z}} .
$$

Integrating over 1000 turns we find that many of the asymmetric features of the evolution of the beam can be reproduced. A comparison between the simulation and actual beam motion is shown in Figs. 8 and 9.

Although much of the structure of the beam envelope evolution has been captured by this simple model, there
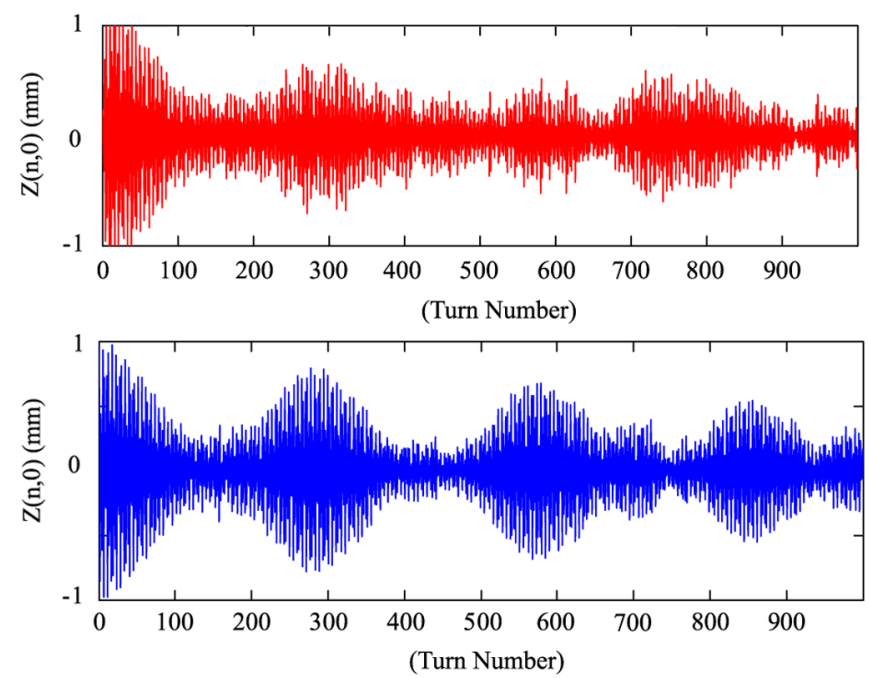

FIG. 10. (Color) Plot of measured and simulated $Z(n, \tau)$ versus $n$ turns. Shown is the transverse (horizontal) turn-by-turn data of center ( $\tau=0$ nsecs) after a $1 \mathrm{~mm}$ kick. Actual turn-by-turn data (top) compared with 1000 particle simulation with resistive wall wake $W_{1 \perp}=12 \mathrm{~cm}^{1.5}$ and chromaticity $\xi=3.733$ and $\sigma_{\tau}=$ 3 nsecs. 
remain a few significant discrepancies. First, the overall decoherence time is not accurately represented by this model and, second, the envelope has significant mismatch at the center of the bunch as is shown in Fig. 10.

\section{EXTENDING MODEL TO 2ND ORDER CHROMATICITY}

We now extend the model to include second order chromaticity. This leads to a simple modification of Eq. (15) and (16), to include the phase terms given in Eq. (11):

$$
z 2_{i p 1}=r_{i p 1}^{2}\left(\frac{\phi_{i p 1}}{2}-\frac{\sin \left(2 \phi_{i p 1}\right)}{4}\right)
$$

Here $z 2_{i p 1}$ is the 2nd order part of the phase and $\phi_{i p 1}=$ $\omega_{s} s / c+\phi$. So now Eq. (15) becomes

$$
\begin{aligned}
d A\left[z_{i p 1}(n), n\right]= & i w r \sum_{i p 2}^{N_{p}} A\left[z_{i p 2}(n), n\right] \\
& \times e^{(-i \chi / c)\left[z_{i p 1}(n)-z_{i p 1}(0)-z_{i p 2}(n)+z_{i p 2}(0)\right]} \\
& \times e^{-i\left(\chi_{2} / c^{2}\right)\left[z z_{i p 1}(n)-z 2_{i p 1}(0)-z 2_{i p 2}(n)+z 2_{i p 2}(0)\right]} \\
& \times W_{0 \perp}\left[z_{i p 1}(n)-z_{i p 2}(n)\right]
\end{aligned}
$$

and Eq. (16) becomes
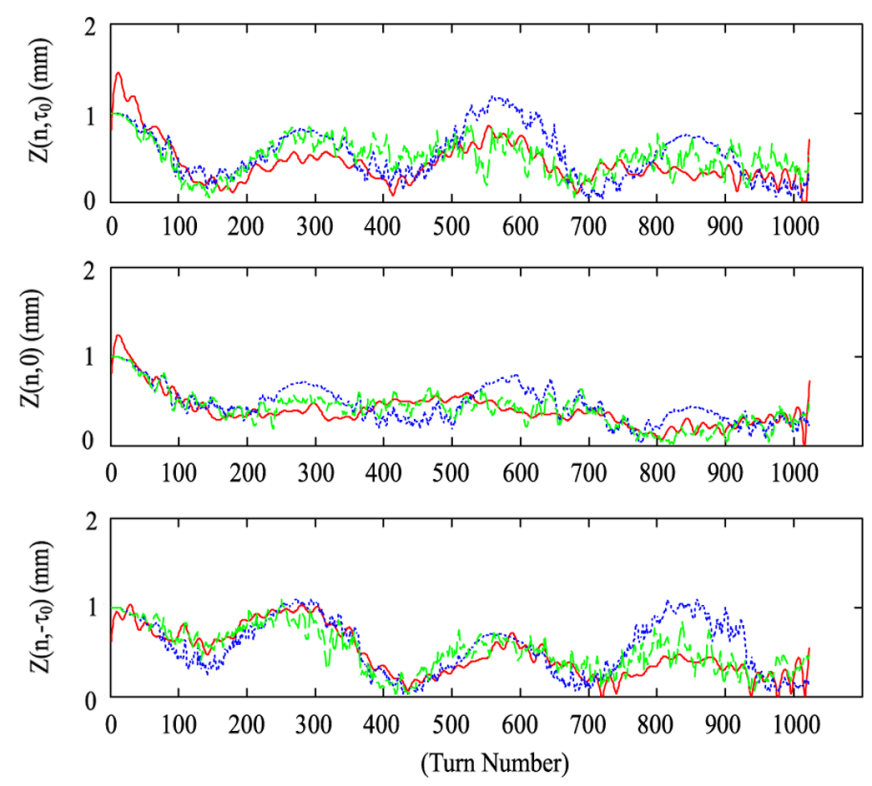

FIG. 11. (Color) Plot of measured and simulated envelope of $Z(n, \tau)$ versus $n$ turns. Shown is the transverse (horizontal) envelope of turn-by-turn data of head (top), center (middle), and tail (bottom) after a $1 \mathrm{~mm}$ kick. The head and tail are 4 nsecs $\left(\tau_{0}=4\right.$ nsecs) away from the bucket center. Shown is the actual envelope data (red trace) overlaid with 1000 particle simulation with resistive wall wake $W_{1 \perp}=12 \mathrm{~cm}^{1.5}$ and chromaticity $\xi=$ 3.733 and $\sigma_{\tau}=3 \mathrm{~ns}$ only (blue trace). Simulations with the addition of second order chromaticity of $\xi^{\prime}=3000$ (green trace) are also overlaid.

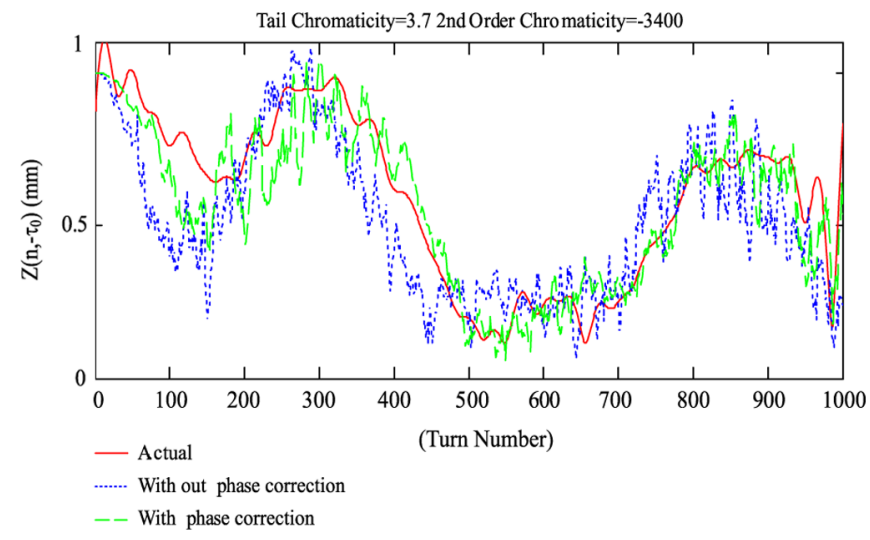

FIG. 12. (Color) Plot of measured and simulated envelope of $Z(n, \tau)$ versus $n$ turns. Shown is the (vertical) transverse envelope of the turn-by-turn data for the tail after a $1 \mathrm{~mm}$ kick. The tail is $4 \mathrm{~ns}$ away from the bucket center ( $\left.\tau_{0}=4 \mathrm{nsecs}\right)$. The red trace is actual vertical data, blue simulation with resistive wall wake $W_{1 \perp}=19 \mathrm{~cm}^{1.5}$, chromaticity $\xi=3.7$, bunch length $\sigma_{\tau}=3 \mathrm{~ns}$, second order chromaticity of $\xi^{\prime}=-3400$ only. The green trace shows the effect of adding Eq. (21) correction to the synchrotron phase.

$$
\begin{aligned}
A b(z, n)= & \sum_{i p}^{N_{p}} A\left[z_{i p}(n), n\right] e^{(-i \chi / c)\left[z_{i p}(n)-z_{i p}(0)\right]} \\
& \times e^{\left(-i \chi_{2} / c^{2}\right)\left[z z_{i p}(n)-z 2_{i p}(0)\right]} \Theta\left[\left|z-z_{i p}(n)\right|-L\right] .
\end{aligned}
$$

Here we define a second order head-tail phase amplitude term, $\chi_{2}=\frac{\omega_{0} \omega_{s} \xi^{\prime}}{2 \eta^{2}}$. The results of simulations with 2 nd order chromaticity and resistive wall wakefield are shown in Fig. 11, where now with second order chromaticity of about 3000 we can account for both the decoherence time and the envelope structure at the bunch center.

This level of 2 nd order chromaticity compares with measured chromaticity on the order of 1500 units (measured on a separate occasion). Checking the vertical plane as can be seen in Fig. 12, the best fit yields -3400 units. This compares with -1700 (measured on a separate occasion) for the measured values.

\section{INCLUSION OF SYNCHROTRON FREQUENCY SPREAD}

Without too much difficulty, it is possible to further extend our model to include the effective synchrotron frequency spread for particles oscillating in the outer part of the rf bucket. This effect can be approximated by allowing our phase to increment to shift with radial amplitude as

$$
\delta \phi_{i p} \approx-k_{r f}^{2} r_{i p}^{2} / 16
$$

The inclusion of this effect shifts the coherence temporal pattern on the outer edges of the bunch allowing an even better fit to our actual data as can be seen in Fig. 12. In this 
figure data from the vertical plane is shown. The fit is clearly improved with inclusion of the 2 nd order synchrotron frequency shift.

\section{FITTING BEAM PARAMETERS TO MODEL}

With a model which appears to represent the main features of the longitudinally sliced wave form, an attempt can be made to use fits to the actual data to extract beam parameters. We accomplished these fits by precomputing scans varying wakefield strength, chromaticity, and 2nd order chromaticity, then picking the minimum $\hat{\chi}^{2}$ value where we define

$$
\hat{\chi}^{2}=\sum \frac{\left(\hat{Z}_{\text {meas }}-\hat{Z}_{\mathrm{fit}}\right)^{2}}{\sigma^{2}}
$$

Here we use a standard deviation of $\sigma=0.18 \mathrm{~mm}$. For these fits $\chi^{2}$ values below 9500 units represent a maximum likelihood that the model is valid and 10000 units is 50\% likelihood.

This approach does require significant computation time up front to generate the scans but once calculated can be reused to fit other data. We used a simple program written in MATHCAD but a short script should work as well. The actual fitting process required only a few minutes. In this way online computation of first and second order chromaticity with resistive wall strength is feasible.
Of course with this approach the resolution of the beam parameters is limited by the precomputed parameter step size and range. Since current measurements of linear chromaticity typically have errors of about $+/-1$ unit and 2nd order $+/-100$ units, we chose a step size of about 0.3 and 1000 which in terms of $\chi$ and $\chi_{2}$ are both 0.1. We chose less precision for the 2 nd order since we did not have any very accurate measurements of this value and for calculation time considerations. We processed several sets of data taken over a $3 \mathrm{yr}$ period of the Tevatron's life, covering the period before the application of the liner on the F0 Lambertson magnet and after. Using our gross fitting method, one can see in Tables I and II, if we ignore the outliers, an overall reduction in the strength of the transverse wake function coefficient for the resistive wall wake, falling from $30-12 \mathrm{~cm}^{-1.5}$ in the horizontal plane and from $34-16 \mathrm{~cm}^{-1.5}$ in the vertical. In terms of effective impedance, this represents a reduction from $2.8-1.0 \mathrm{M} \Omega / \mathrm{m}$ and $3.0-1.5 \mathrm{M} \Omega / \mathrm{m}$ in the horizontal and vertical planes, respectively (at $100 \mathrm{MHz}$ ) which is consistent with previous estimates of the impact of the liner [8] which claimed a reduction from $2.4-1 \mathrm{M} \Omega / \mathrm{m}$.

Over the first 1000 turns we fit the central 10 points of the bunch spanning 4 nsecs. Beyond 10 points the quality of the signal deteriorated with larger chromaticity.

The goodness of the fits depended on the level of linear chromaticity because the signal to noise for the experiment would fall off with chromaticity. This is due to the nature of

TABLE I. Horizontal fits to turn-by-turn data.

\begin{tabular}{|c|c|c|c|c|c|}
\hline T:CXINJ & $N$ particles $\left(10^{11}\right)$ & $\xi_{x} \pm 1$ & $\xi_{x}^{\prime} \pm 10^{3}$ & $W_{1 \perp}\left(1 / \mathrm{cm}^{1.5}\right)$ & $\chi^{2}$ \\
\hline \multicolumn{6}{|c|}{ Data taken November 27, 2003 (before liner install) } \\
\hline 33 & $<1.11$ & 3.425 & 4000 & $19>$ & 4940 \\
\hline 37 & $<1.11$ & 5.293 & 4000 & $32>$ & 5300 \\
\hline 39 & $<1.11$ & 5.604 & 4000 & $38>$ & 6600 \\
\hline 41 & 1.11 & 9.651 & 6000 & 58 & 6000 \\
\hline 37 & 2.26 & 6.227 & 5000 & 28 & 6600 \\
\hline 39 & 2.26 & 7.161 & 5000 & 30 & 4500 \\
\hline 41 & 2.26 & 9.028 & 6000 & 30 & 3300 \\
\hline \multicolumn{6}{|c|}{ Data taken April 15, 2003 (before liner install) } \\
\hline 30 & 1.14 & 0.9 & 2000 & 30 & 3100 \\
\hline \multicolumn{6}{|c|}{ Data taken August 22, 2006 (after liner install) } \\
\hline 44 & 2.30 & 6 & 1000 & 12.5 & 4100 \\
\hline 42 & 2.30 & 4 & 1000 & 12.5 & 3318 \\
\hline 41 & 2.30 & 3.425 & 1000 & 12.5 & 2612 \\
\hline 40 & 2.30 & 2.5 & 0 & 12.5 & 2354 \\
\hline 39 & 2.30 & 2.179 & 0 & 12.5 & 2516 \\
\hline \multicolumn{6}{|c|}{ Data taken August 24, 2006 (after liner install) } \\
\hline 39 & 2.44 & 1.8 & 1000 & 12 & 247 \\
\hline 41 & 2.44 & 3.425 & 2000 & 12 & 494 \\
\hline 42 & 2.44 & 4.67 & 3000 & 12 & 806 \\
\hline 43 & 2.44 & 5.6 & 4000 & 12 & 3100 \\
\hline 44 & 2.44 & 8 & 0 & 16 & 6300 \\
\hline
\end{tabular}


TABLE II. Vertical fits to turn-by-turn data.

\begin{tabular}{|c|c|c|c|c|c|}
\hline $\mathrm{T}: \mathrm{CYINJ}$ & $N$ particles $\left(10^{11}\right)$ & $\xi_{y} \pm 1$ & $\xi_{y}^{\prime} \pm 10^{3}$ & $W_{1 \perp}\left(1 / \mathrm{cm}^{1.5}\right)$ & $\chi^{2}$ \\
\hline \multicolumn{6}{|c|}{ Data taken April 15, 2003 (before liner install) } \\
\hline 33 & 1.59 & 11 & -7666 & 40.7 & 13007 \\
\hline 35 & 1.61 & 8.7 & -5000 & 40.2 & 6618 \\
\hline 31.5 & 1.26 & 5 & -5000 & 44 & 5871 \\
\hline 28 & 1.00 & 2.4 & 1000 & 29 & 1681 \\
\hline 28 & 1.80 & 2.4 & -1000 & 24 & 3320 \\
\hline 28 & 1.85 & 3.3 & -4000 & 33 & 3394 \\
\hline 28 & 0.86 & 2.1 & -4000 & 34 & 6328 \\
\hline 30 & 1.69 & 3.3 & -4000 & 18 & 3353 \\
\hline 26 & 1.82 & 1.2 & -2000 & 12 & 2529 \\
\hline 32 & 1.87 & 8.7 & -5000 & 35 & 6755 \\
\hline \multicolumn{6}{|c|}{ Data taken August 24, 2006 (after liner install) } \\
\hline 25 & 2.55 & 5 & -4000 & 25 & 6823 \\
\hline 24 & 2.55 & 4.35 & -3000 & 19 & 5500 \\
\hline 23 & 2.55 & 3.425 & -3000 & 16 & 3567 \\
\hline 22 & 2.55 & 2.802 & -2000 & 16 & 6610 \\
\hline 22.5 & 2.55 & 3.425 & -2000 & 19 & 5870 \\
\hline
\end{tabular}

the chromaticity sextupole magnets whose contribution to 2nd order chromaticity increases with field strength. Thus, with higher 2 nd order chromaticity more damping was present.

This increase in noise level was also present in the simulation; as 2nd order chromaticity and wakefield strength were increased, the noise of our results increased using only 1000 macroparticles. A higher number of macroparticles were needed to lower the noise levels of the simulation results and required longer simulation times. Because of time considerations, we chose to use only 1000 macroparticles. This resulted in the quality of the fits falling off above 6 units of machine chromaticity. The usable signal across the bunch would fall off from the ends of the bunch as chromaticity increased. This had the general effect of blurring the effect of the short range wake with that of 2 nd order chromaticity since the distinguishing features are evident in ends of the bunch. As a result, those fits with chromaticity above 6 units would often settle in a false $\hat{\chi}^{2}$ minimum mixing 2nd order chromatic effects with wakefield and vice versa. On the lower end of the chromaticity values, this problem of distinguishing 2nd order chromaticity from short range wakefield effects was evident due to the fact that a very small level of 2 nd order chromaticity can "look like" the effects of a short range wakefield. This problem can be seen reflected in our data as an undervaluing of the short range wakefield strength at low chromaticities and overvaluing for high chromaticities.

The data set taken on April 15th also had problems since the horizontal chromaticity was not kept constant and it is unclear what impact the high horizontal chromatic sextupole would have on 2 nd order chromaticity in the vertical plane. Since we only fit the beam envelope, it would seem that fitting the phase may help above 6 units of chromatic- ity and minimize the effect of the redundant minimums. In fact, a comparison of the phase estimated chromaticity with the beam envelope estimated chromaticity demonstrated a growing inconsistency with chromaticities greater than 6 units. The phase data appeared to be generally more consistent with the machine sextupole current settings than the envelope data above six units of chromaticity.

Our experience suggests that the best approach to apply this method would be to first independently measure linear and second order chromaticity and then fit the wakefield coefficient, or conversely, once the strength of the short range wakefield is determined to fit linear and 2nd order chromaticity.

\section{CONCLUSION}

Using a simple multiparticle simulation, we have been able to recover the outstanding features of the single bunched beam evolution after the application of a kick. Comparisons with this model allowed us to fit both the strength of the associated wakefield due to resistive wall and deliver values for 1 st and 2 nd order chromaticity. Using a database of precomputed scans can permit the fitting of either all three parameters, linear chromaticity, 2nd order chromaticity, and resistive wall wakefield strength, or more accurately measuring two or more parameters and fitting the third providing a robust method of measuring the relevant beam parameters during operation.

\section{ACKNOWLEDGMENTS}

We are particularly grateful to Alexey Burov for his advice and for supplying us with a basic $\mathrm{C}++$ simulation code. This code was built upon to develop the simulations shown here. 
[1] P. M. Ivanov et al., in Proceedings of the Particle Accelerator Conference, Portland, OR, 2003 (IEEE, New York, 2003), p. 3062.

[2] P.M. Ivanov et al., Proceedings of Particle Accelerator Conference (PAC 05), Knoxville, Tennessee, 2005, p. 2714.

[3] R. Jones and H. Schmickler, CERN Accelerator School Intermediate Accelerator Physics DESY, Zeuthen, Germany, 2003, http://doc.cern.ch/yellowrep/2006/2006002/p75.pdf.
[4] S. Fartoukh and R. Jones, LHC Project Report No. 602, 2002.

[5] A. W. Chao, Physics of Collective Beam Instabilities in High Energy Accelerators (John Wiley and Sons, New York, 1993), pp. 147-149.

[6] M. Xiao et al., in Ref. [1], p. 1775.

[7] A. W. Chao, in Ref. [5], pp 58-59.

[8] P. M. Ivanov, F0 Lambertson liner review, internal FNAL document (2003). 